\title{
Motion of red blood cells in a glass microchannel: a global optimization approach
}

\author{
Diana Pinho, Ana I. Pereira and Rui Lima \\ Polytechnic Institute of Bragança, Portugal
}

\begin{abstract}
.
In this work we characterized the behavior of red blood cell motion through a glass microchannel. In this study we consider the radial displacement of forty red blood cells and use different functions to approximate the radial displacement of each of them, by means of global optimization using stretched simulated annealing method. Some numerical results are shown.

Keywords: Numerical Optimization. Red blood cells. Global Optimization

PACS: $02.60 . \mathrm{Pn}$
\end{abstract}

\section{INTRODUCTION}

Blood is a fluid composed of a suspension of cells, proteins and ions in plasma. In normal blood, three types of cells comprise about $46 \%$ of its volume. These cells are the red blood cells (also known as erythrocytes), white blood cells (also known as leukocytes) and platelets (also known as thrombocytes). The main function of the blood is to transport oxygen, nutrients, waste products and heat throughout the body. The circulating blood cells suspended in the plasma are:

- Red blood cells (RBCs) - which are the most abundant blood cells and contain hemoglobin.

- Platelets - which are essential for cellular blood clotting.

- White blood cells - which are nucleated cells that represent the major defence mechanism against infections.

Approximately half of the volume of blood is composed of red blood cells (RBC), which is believed to strongly influence the blood flow properties. Blood flow in microvessels depends strongly on the motion, deformation and interaction of RBCs. Hence detailed knowledge on the motion of individual RBCs flowing in microchannels is essential to provide a better understanding on the blood rheological properties and disorders in microvessels .

The paper is organized as follows. In Section 2 we describe the red blood cells motion and the problem formulation in a optimization context. The Section 3 present the stretched simulated annealing method, and the Section 4 contains some numerical experiments. We conclude the paper in the last section.

\section{RED BLOOD CELLS MOTION}

In the microcirculation, the flow behavior of RBCs plays a crucial role in many physiological and pathological phenomena. For example, the random-like transverse motion and rotation of RBCs in shear flow is believed to play an important role in thrombogenesis. However, the role of RBCs in the mass transport mechanism of cells and proteins to the thrombus is still not completely understood $[7,18,11]$. As a consequence, many studies have been performed on both the rheological and microrheological behavior of RBCs flowing through glass capillaries [2,11]. It is then on the cells that addresses this problem is to try to bring a light its movement along the micro channel. The data provided to study the RBC displacement was obtained from [8], where the radial displacement of RBCs was obtained by a confocal micro-PTV system [9]. In this study the RBC dispersion coefficient was measured by using the following equation :

$$
d_{y}(t)=\frac{\left(R_{y}(t)-R_{y}(0)\right)^{2}}{2 t}
$$




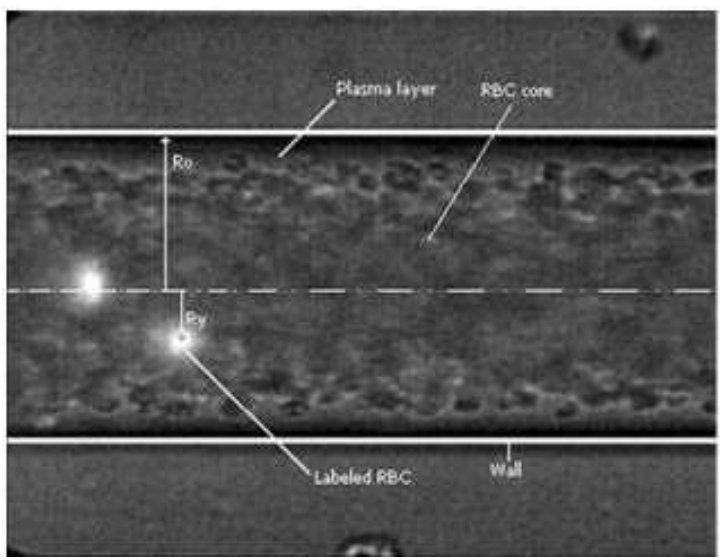

FIGURE 1. Coordinate system in the middle plane of a $100 \mu \mathrm{m}$ capillary. The image contains both halogen and laser light, which enables visualization of both labeled and non-labeled RBCs.

where $R_{y}(t)-R_{y}(0)$ is the radial distance traveled by individual RBC, in the position $y$, over some time interval of length $t[8]$.

The time set consider was $[0,0.4]$, and we fix 20 measurements for each RBC over a glass microchannel. The glass microchannel has a diameter of $100 \mu \mathrm{m}$.

The objective of this paper is to obtain a better approximation of the obtained data through global optimization. We considerer four different functions (polynomials, exponential and logarithmic) defined as: $h_{1}(t)=x_{3} t^{2}+x_{2} t+x_{1}$, $h_{2}(t)=x_{4} t^{3}+x_{3} t^{2}+x_{2} t+x_{1}, h_{3}(t)=x_{2} e^{x_{1} t}$ and finally $h_{4}(t)=x_{2} \ln \left(x_{1} t\right)$ considering $x_{1}>0$.

The problem can be formulated as

$$
\min f(x) \equiv \sum_{i=0}^{n}\left(y_{i}-h_{k}\left(t_{i}\right)\right)^{2}
$$

were $x \in \mathbb{R}^{p}$ and the data $\left(t_{i}, d_{y}^{i}\right)$, for $i=1, \ldots, 20$, it was obtained from all the experiences.

\section{OPTIMIZATION TECHNIQUE}

To solve the problem (1) we consider the Stretched Simulated Annealing (SSA) method [15].

The SSA algorithm is capable of locating global solutions of problem (1) combining the simulated annealing method with local applications of the function stretching technique and a local search [13, 14].

Consider the functions

$$
\begin{gathered}
\bar{g}(t)=g(t)-\frac{\delta_{1}}{2}\|t-\bar{t}\|(\operatorname{sign}(g(\bar{t})-g(t))+1), \\
\widetilde{g}(t)=\bar{g}(t)-\frac{\delta_{2}}{2} \frac{\operatorname{sign}(g(\bar{t})-g(t))+1}{\tanh (\mu(\bar{g}(\bar{t})-\bar{g}(t)))}
\end{gathered}
$$

where $\delta_{1}, \delta_{2}$ and $\mu$ are positive constants.

At each iteration $k$, the SSA algorithm solves, using the simulated annealing variant, the following global optimization problem:

$$
\max _{t \in T} \Phi_{k}(t) \equiv\left\{\begin{array}{cc}
g(t) & \text { if } k=1 \\
w(t) & \text { if } k>1
\end{array}\right.
$$

where the function $w(t)$ is defined as

$$
w(t)= \begin{cases}\widetilde{g}(t) & \text { if } t \in V_{\varepsilon}\left(\bar{t}^{i}\right) \\ g(t) & \text { otherwise }\end{cases}
$$


and $\bar{t}^{i}(i=1,2, \ldots, \bar{m})$ denotes a previously found global maximizer. $V_{\varepsilon}\left(\bar{t}^{i}\right)$ represents a neighborhood of $\bar{t}^{i}$, with ray $\varepsilon$, $\bar{m}$ is the number of previously found global solutions of (1) and $\widetilde{g}$ is the function defined in (3).

The SSA algorithm resorts in a sequence of global optimization problems whose objective functions are the original $g$, in the first iteration, and the transformed $w$ in the subsequent iterations. As the function stretching technique is only applied in a neighborhood of an already detected global maximizer, the simulated annealing global algorithm is able to identify the other global maximizers that were not yet found.

\section{NUMERICAL RESULTS}

The proposed algorithm was implemented on a Pentium II Celeron $466 \mathrm{Mhz}$ with 64Mb of RAM, in the C programming language and connected with AMPL [3] to provide the coded problems. AMPL is a mathematical programming language that allows the codification of optimization problems in a powerful and easy to learn language. AMPL also provides an interface that can be used to communicate with a solver.

Details of the selected cells are listed in the table, where Cell refers to the cell number, $\lambda$ is the cell initial position in microchannel, $f_{h_{i}}$ the optimal value of objective function considering $h_{i}$, for $i=1, . .4$.

TABLE 1. Numerical Results obtained using SSA method

\begin{tabular}{lccccc}
\hline Cell & $\lambda$ & $f_{h_{1}}$ & $f_{h_{2}}$ & $f_{h_{3}}$ & $f_{h_{4}}$ \\
\hline 5 & 8.74 & $9.6579 E^{-16}$ & $5.5268 E^{-17}$ & $3.7426 E^{-15}$ & $4.9680 E^{-15}$ \\
10 & 15.37 & $6.7465 E^{-17}$ & $4.7784 E^{-19}$ & $1.7108 E^{-16}$ & $2.3731 E^{-17}$ \\
24 & 66.25 & $7.9289 E^{-18}$ & $5.0578 E^{-18}$ & $7.4643 E^{-16}$ & $2.5057 E^{-16}$ \\
34 & 74.66 & $3.0965 E^{-17}$ & $3.6518 E^{-18}$ & $2.0836 E^{-15}$ & $1.9384 E^{-15}$ \\
36 & 78.99 & $3.7209 E^{-17}$ & $3.4104 E^{-18}$ & $4.3507 E^{-16}$ & $4.1258 E^{-16}$ \\
40 & 81.74 & $1.3687 E^{-17}$ & $9.9042 E^{-18}$ & $1.4586 E^{-15}$ & $6.2699 E^{-16}$ \\
\hline
\end{tabular}

We can conclude that the approximations obtained by the software SSA are similar that were obtained by Matlab optimization toolbox (polyfit and fminsearch).

Calculating the results of Table 1, we can see that the polynomial of degree three was a better approximation to the movement of the cells in the microchannel, since gives a less objective function value.

\section{CONCLUSIONS}

In this work we consider the radial displacement of RBCs. The obtained data was approximated by four different types of functions, the sense of least squares. To solve the unconstrained optimization problem we consider the SSA method.

From this study, we may conclude that, in general, the function that better approximate the data is $h_{2}$. In future work we will consider different type of functions.

An on going study to obtain more detailed quantitative measurements of the blood flow behavior through a glass microchannel is currently under way.

\section{REFERENCES}

1. D. P. Bertsekas, Constrained Optimization and Lagrange Multiplier Methods, Academic Press, 1982.

2. S. Chien, S. Usami and R. Skalak, Blood flow in small tubes, In Handbook of Physiology-The Cardiovascular System IV, pp. $217-249$ (1984).

3. R. Fourer, D. Gay, and B. Kernighan, A modeling language for mathematical programming, Management Science, 36, no. 5, 519-554 (1990).

4. D. Lominadze and G . Mchedlishvili,Red Blood Cell Behavior at Low Flow Rate in Microvessels, Microcirculation Research Center, I. Beritashvili Institute of Physiology, (1998).

5. L. Ingber, Very fast simulated re-annealing, Mathematical and Computer Modelling, 12, 967-973 (1989).

6. T. Ishikawa and T. Pedley, Diffusion of swimming model microorganisms in a semi-dilute suspensions, Journal of Fluid Mechanics, 588, 437-462, (2007). 
7. H. Goldsmith and V. Turitto, Rheological aspects of thrombosis and haemostasis: basic principles and applications. ICTH-Report-Subcommittee on Rheology of the International committee on thrombosis and haemostasis. Thrombosis and Haemostasis, 55, 415-435 (1986).

8. R. Lima, T. Ishikawa, Y. Imai, M. Takeda, S. Wada and T. Yamaguchi, Radial dispersion of red blood cells in blood flowing through glass capillaries: The role of hematocrit and geometry, Journal of Biomechanics, 41, 2188-2196 (2008).

9. R. Lima, M. Nakamura, T. Omori, T. Ishikawa, S. Wada, and T. Yamaguchi, Microscale Flow Dynamics of Red Blood Cells in Microchannels: An Experimental and Numerical Analysis, J.M.R.S. Tavares, R.M.N. Jorge (eds.), Advances in Computational Vision 203 and Medical Image Processing, Computational Methods in Applied Sciences, 13, Chapter 12, 203-219 (2008).

10. MathWorks, Optimization Toolbox User's Guide, 1990-2009.

11. G. Mchedlishvili and N. Maeda, Blood flow structure related to red cell flow: a determination of blood fluidity in narrow microvessels, Japanese Journal of Physiology, 51, 19-30 (2001).

12. H. Miyazaki, T. Miyazaki, Formation and destruction of primary thrombi under the influence of blood flow and von willebrand factor analysed,D.E.M. Biorheology, 40, 265-272 (2003).

13. K. Parsopoulos, V. Plagianakos, G. Magoulas, and M. Vrahatis, Objective function stretching to alleviate convergence to local minima, Nonlinear Analysis 47, 3419-3424 (2001).

14. K. Parsopoulos and M. Vrahatis, Recent approaches to global optimization problems through particle swarm optimazition, Natural Computing 1, 235-306 (2002).

15. A. I. P. N. Pereira and E. M. G. P. Fernandes, A reduction method for semi-infinite programming by means of a global stochastic approach, Optimization, 58, 713-726 (2009).

16. A. Pries,D. Neuhaus, P. Gaehtgens,Blood viscosity in tube flow: dependence on diameter and hematocrit, American Journal of Physiology 263, H1770-H1778, (1992).

17. T. Shiga, N. Maeda and K. Kon, Erythrocyte rheology. Critical Review in Oncology/Hematology, 10, 9-48 (1990).

18. D. Ku and D. Wootton, Fluid mechanics of vascular systems, diseases, and thrombosis. Annual Review of Biomedical Engineering, 1, 299-329 (1999). 